\title{
Comparison of Intraoperative Parameters and Perioperative Complications of Retroperitoneal and Transperitoneal Approaches to Laparoscopic Partial Nephrectomy: Support for a Retroperitoneal Approach in Selected Patients
}

\author{
KATHLEEN KIERAN, M.D., JEFFREY S. MONTGOMERY, M.D., STEPHANIE DAIGNAULT, M.S., \\ WILLIAM W. ROBERTS, M.D., and J. STUART WOLF, JR., M.D.
}

\begin{abstract}
Background and Purpose: Retroperitoneal laparoscopy (RP) may have some intrinsic advantages over transperitoneal laparoscopy (TP) in certain patients undergoing partial nephrectomy. We reviewed our experience with RP and TP partial nephrectomy to identify differences in intraoperative and postoperative parameters.

Patients and Methods: The records of 72 patients (45 TP, $27 \mathrm{RP}$ ) undergoing laparoscopic partial nephrectomy without hand assistance between January 2003 and August 2005 were reviewed. The two groups were similar demographically; tumors were smaller in RP patients $(2.1 v 2.7 \mathrm{~cm} ; P=0.03)$, and the RP approach was used more frequently on right kidneys $(70.4 \% v 37.8 \% ; P=0.01)$.

Results: The operative time (mean $160 v 192$ minutes; $P=0.008$ ) and length of stay (LOS; median 1.0 days [range 1-10 days] $v$ 2.0 days [range 1-64 days]; $P=0.001)$ were shorter in RP patients. Rates of collecting system entry $(22 \% v 38 \%)$, positive-margin rate $(0 \% v 6.7 \% ; P=0.29)$, and complications $(19 \% v 22 \% ; P=$ 0.77) were similar in RP and TP patients. Hemorrhage was the most common complication in both groups. Bowel-related complications occurred in three TP patients, but in no RP patients. Overall, the median estimated blood loss (EBL) was lower in RP patients $(100 \mathrm{~mL}$ [range $25-3500 \mathrm{~mL}$ ] $v 225 \mathrm{~mL}$ [range 25-1900 $\mathrm{mL}$; $\boldsymbol{P}=\mathbf{0 . 0 6}$ ). Among patients with complications, EBL was similar in both groups (median $325 \mathrm{~mL}$ [50-1500 $\mathrm{mL}] v 200 \mathrm{~mL}[50-3500 \mathrm{~mL}]$ for $\mathrm{RP}$ and $\mathrm{TP} ; P=0.86$ ).

Conclusions: The RP approach reduces operative time, LOS, and some types of complications without compromising the quality of tumor resection. Complications in the retroperitoneal space are not associated with higher EBL. Anatomic considerations and surgeon experience may improve outcomes.
\end{abstract}

\section{INTRODUCTION}

$\mathbf{T}$ HE RENAL MASSES being diagnosed today are smaller and more likely to be asymptomatic than those diagnosed 10 years ago. ${ }^{1}$ This, as well as the evolving understanding that tumor extirpation and renal parenchymal preservation are not mutually exclusive, have popularized partial and laparoscopic nephrectomy techniques. Partial nephrectomy enables complete removal of the tumor with pathologic interpretation and assessment of margin status while preserving normal renal parenchyma and without compromising neoplastic $\operatorname{control}^{2,3}$; lap- aroscopic techniques for both radical and partial nephrectomy have been shown to be equivalent to or better than open surgery with regard to recovery time, length of stay (LOS), and cost. $^{4-7}$ Although there are case reports of port-site recurrences of neoplasm, ${ }^{8}$ larger studies have not found this to be a significant risk, and present research suggests that cancer control is similar with open and laparoscopic techniques. ${ }^{6,9-11}$

One limitation of the laparoscopic transperitoneal (TP) approach to partial nephrectomy is the difficult dissection of posterior or posterolateral tumors. ${ }^{12,13}$ The retroperitoneal (RP) approach offers a more direct route of access to the kidney and 


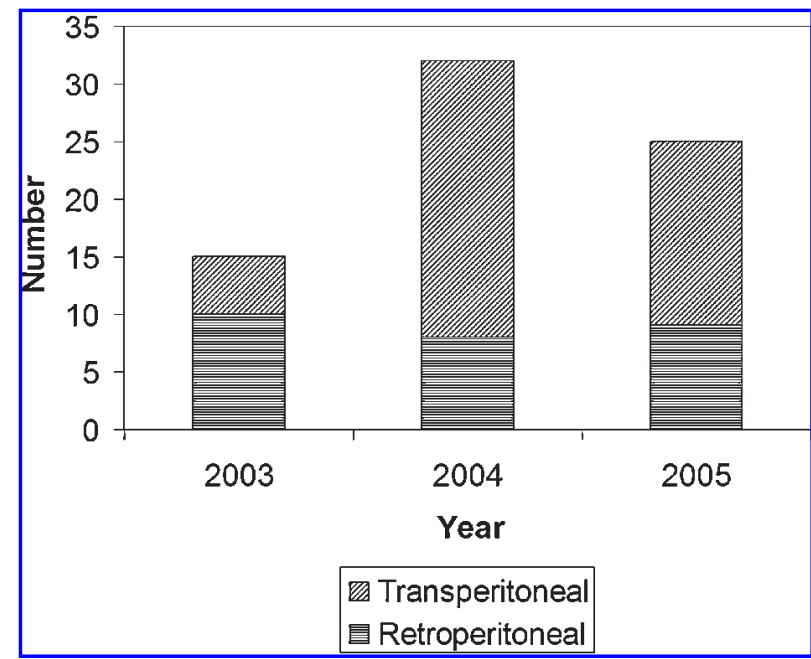

FIG. 1. Trends in use of TP and RP approaches to partial nephrectomy at University of Michigan from January 2003 to August 2005 (excludes hand-assisted cases).

posterior tumors and may require less dissection than the transperitoneal approach. ${ }^{14}$ Gastrointestinal complications account for much of the morbidity of TP procedures: bowel injuries are difficult to identify intraoperatively and diagnose postoperatively, and ileus is the foremost reason for early readmission. ${ }^{15-17}$ The limited dissection and visceral manipulation in the RP approach may therefore not only increase operative efficiency but also decrease postoperative recovery time and pain.

Although prior research has suggested that surgical outcomes are similar with the TP and RP approaches to radical nephrectomy and adrenalectomy, ${ }^{18,19}$ there is a dearth of literature comparing the two approaches for the more complex partial nephrectomy. We report our preliminary experience with TP and RP laparoscopic partial nephrectomy, with specific emphasis on differential intraoperative and perioperative outcomes.

\section{PATIENTS AND METHODS}

Institutional Review Board approval was granted prior to the start of this study. Using the University of Michigan Laparoscopy/Endourology Database, we identified all patients who had undergone either TP or RP laparoscopic partial nephrectomy by a single surgeon (J.S.W.) for treatment of a renal mass suspected of being a neoplasm between January 2003 and August 2005. The choice of technique was at the surgeon's discretion. Generally, the RP approach was used for posterior masses and the TP approach for anterior and polar masses. Patients undergoing planned hand-assisted laparoscopic surgery or ablation rather than complete excision (e.g., laparoscopic radiofrequency ablation) were excluded from review.

A retrospective medical record review was conducted of laparoscopic partial nephrectomies carried out between January 2003 and August 2005, with the specific data abstracted including demographics, preoperative lesion characteristics (size, laterality), intraoperative parameters (estimated blood loss [EBL], operative time, renal ischemia time, and need for conversion to alternate procedures), pathologic findings (tumor classification and margin status), LOS, and perioperative complications. A total of 106 patients underwent laparoscopic partial nephrectomy of which 72 (45 TP, $27 \mathrm{RP}$ ) fit the inclusion criteria for the study (Fig. 1). The patients in the two groups were similar with regard to age, sex distribution, preoperative renal function, and final pathology of the renal mass (Table 1). Masses treated with the RP approach tended to be smaller (2.1 $v 2.7 \mathrm{~cm} ; P=0.03)$ and were more likely to be on the right side $(70 \%$ v $21 \% ; P=0.01)$.

Table 1. Patient Demographics and Lesion Characteristics

\begin{tabular}{|c|c|c|c|}
\hline & $\begin{array}{l}\text { Transperitoneal } \\
\qquad(\mathrm{N}=45)\end{array}$ & $\begin{array}{l}\text { Retroperitoneal } \\
\qquad(\mathrm{N}=27)\end{array}$ & $\mathrm{P}$ value \\
\hline Mean age $\pm \mathrm{SD}$ (years) & $59.2 \pm 14.9$ & $57.0 \pm 14.4$ & 0.55 \\
\hline Males/Females (\%) & $69 / 31$ & $48 / 52$ & 0.09 \\
\hline Mean size of mass $\pm \mathrm{SD}(\mathrm{cm})$ & $2.66 \pm 1.20$ & $2.05 \pm 0.84$ & 0.03 \\
\hline Left/Right $(\%)$ & $62.2 / 37.8$ & 29.6/70.4 & 0.01 \\
\hline Intrarenal location $(\%)$ & & & 0.05 \\
\hline Upper pole & 7 (15.6) & $9(33.3)$ & \\
\hline Interpolar & $22(48.9)$ & $6(22.2)$ & \\
\hline Lower pole & $16(35.6)$ & $12(44.4)$ & \\
\hline Mean distance from renal sinus $\pm \mathrm{SD}(\mathrm{cm})$ & $2.14 \pm 4.40$ & $3.74 \pm 5.72$ & 0.26 \\
\hline $\begin{array}{l}\text { Preoperative serum creatinine } \\
\quad(\mathrm{mg} / \mathrm{dL})\end{array}$ & $1.05 \pm 0.24$ & $0.99 \pm 0.30$ & 0.29 \\
\hline Final pathology diagnosis & & & 0.91 \\
\hline \multicolumn{4}{|l|}{ Renal-cell carcinoma } \\
\hline Clear cell & 20 & 11 & \\
\hline Papillary & 12 & 7 & \\
\hline Chromophobe & 2 & 1 & \\
\hline Oncocytoma & 5 & 4 & \\
\hline Angiomyolipoma & 4 & 1 & \\
\hline Benign cyst & 1 & 2 & \\
\hline Nonfunctioning parenchyma & 1 & 0 & \\
\hline
\end{tabular}


Table 2. Operative and Postoperative Characteristics

\begin{tabular}{lccc}
\hline & Transperitoneal & Retroperitoneal & P value \\
\hline $\begin{array}{l}\text { Collecting system/sinus entry (\%) } \\
\text { Postoperative creatinine change } \\
\quad(\mathrm{mg} / \mathrm{dL})\end{array}$ & $17(37.8 \%)$ & $6(22.2 \%)$ & 0.20 \\
$\begin{array}{l}\text { Complications (\%) } \\
\begin{array}{l}\text { Median LOS (days) } \\
\quad(\text { range) }\end{array}\end{array}$ & $0.11 \pm 0.26$ & $0.11 \pm 0.18$ & 0.96 \\
Positive margins $(\%)$ & $2.00(1.0-64.0)$ & $5(18.5)$ & 0.77 \\
\hline
\end{tabular}

Continuous variables were compared with Student's $t$-test (for two groups) or one-way ANOVA (for three or more groups); nonparametric data were analyzed using the Wilcoxon rank test. Fisher's exact test was used to compare categorical variables. All data testing was performed with the Statistical Analysis System (SAS; SAS Institute, Cary, NC) V. 9.1 software. $P$ values represent two-tailed distributions, and findings were considered significant at $P<0.05$.

\section{RESULTS}

Operative parameters and postoperative characteristics are summarized in Table 2 . The operative time was significantly shorter in RP than in TP patients (160 v 192 minutes; $P=$ 0.008), although the renal ischemic times (22.7 v 25.6 minutes; $P=0.32$ ) were similar (Fig. 2). The rate of entry into the collecting system or renal sinus was similar in the two groups (22.2\% RP, 37.8\% TP; $P=0.20$ ). The median LOS was shorter after RP (1.0 days; range 1-10 days) than TP (2.0 days; range $1-64$ days) $(P=0.001)$. Positive margins were not found in the RP group, although this rate was not statistically different from the TP group (3 patients; 6.7\%) $(P=0.29)$. Preoperative renal function, as assessed by serum creatinine concentration (0.99 [RP] $v 1.05[\mathrm{TP}] \mathrm{mg} / \mathrm{dL}$ ) was similar in the two groups, as was the postoperative change in serum creatinine $(+0.11 \mathrm{mg} / \mathrm{dL}$ in both groups; $P=0.96$ ).

A total of 8 patients $(2[7.4 \%]$ RP, $6[13.3 \%]$ TP; $P=1.00)$ were converted intraoperatively to alternate procedures. Conversion was at the surgeon's discretion and was most commonly for control of intraoperative bleeding (1 RP, 3 TP). Other causes of conversion were technical considerations limiting the completion of the planned operation ( $3 \mathrm{TP}$ ) and the need for modification of the technique because of intraoperative pathology findings (1 RP). Overall, EBL was lower in the RP than in the TP group (100 $v 225 \mathrm{~mL} ; P=0.06)$.

Complications (defined as any event necessitating prolonged LOS, significant deviation from treatment plan, or additional treatment) occurred in $19 \%$ of RP and $22 \%$ of TP patients ( $P=$ 0.77). Table 3 lists the types of complications, stratified by approach. In the RP group, there were three patients with one complication, one patient with two complications, and one patient with three complications; in the TP group, there were seven patients with one complication, two patients with two complications, and one patient with four complications. Although retroperitoneal hemorrhage was the most common complication in both groups, the severity of complications was not equiv- alent in the two groups. In particular, gastrointestinal complications (ileus, Ogilvie's syndrome, and colonic ischemia) were more common in TP patients (3/45 [6.7\%] $v 0)$, although this difference was not statistically significant $(P=0.24)$. Patients with and without complications were demographically similar and had comparable lesion characteristics. Among patients without complications, EBL was slightly lower after RP (100 $v 175 \mathrm{~mL} ; P=0.06$; Fig. 3 ). The EBL in patients with complications was similar regardless of whether the RP $(200 \mathrm{~mL})$ or TP $(325 \mathrm{~mL})$ approach had been utilized $(P=0.86)$.

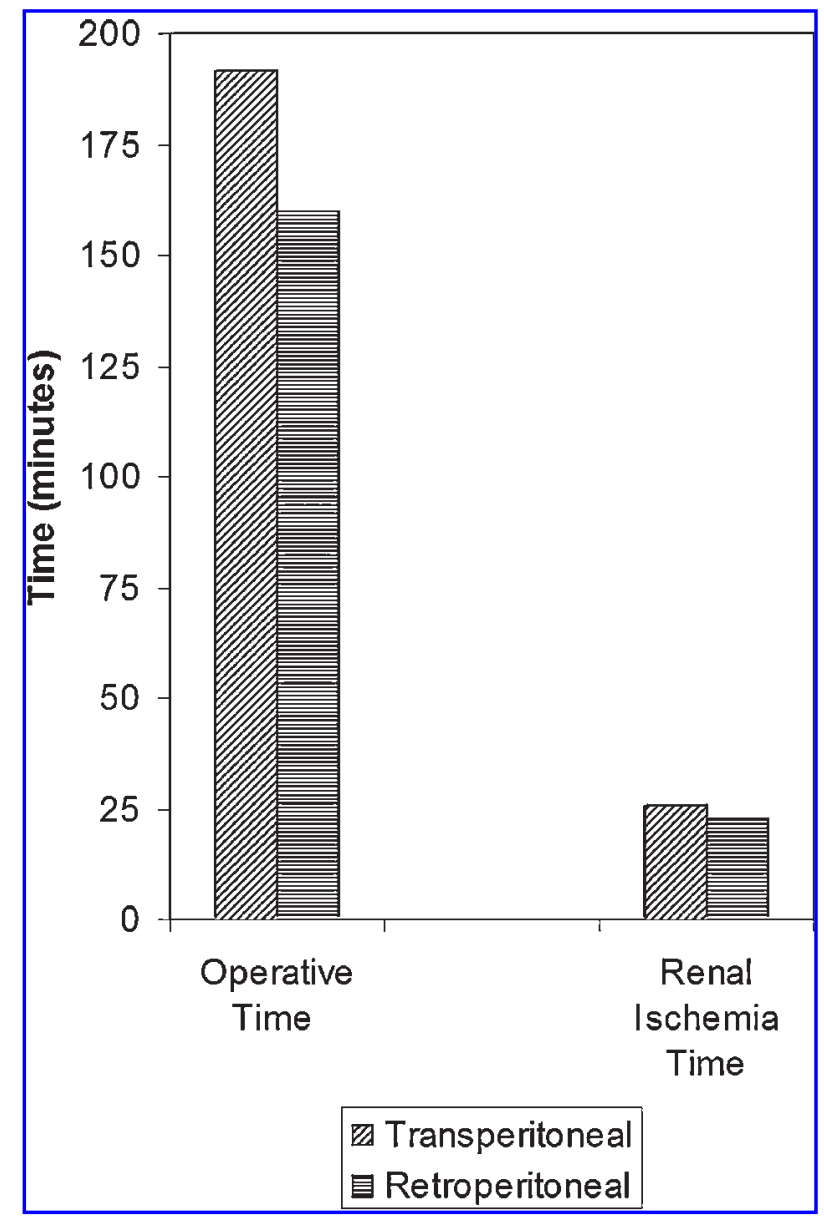

FIG. 2. Operative time is longer with TP approach, whereas ischemia time is similar. 
Table 3. Complications Stratified by Operative Approach

\begin{tabular}{lcc}
\hline & Transperitoneal & Retroperitoneal \\
\hline Retroperitoneal hemorrhage & 6 & 3 \\
Pneumonia & 1 & 1 \\
Readmission for pain control & 2 & 0 \\
Ogilvie's syndrme & 1 & 0 \\
Colonic ischemia & 1 & 0 \\
Respiratory failure & 1 & 0 \\
Arteriovenous fistula & 1 & 0 \\
Sepsis & 1 & 0 \\
Prolonged ileus & 1 & 0 \\
Atrial fibrillation & 0 & 1 \\
Avulsion of renal vein & 0 & 1 \\
Mental status changes & 0 & 1 \\
Corneal abrasion & 0 & 1 \\
\hline
\end{tabular}

\section{DISCUSSION}

In our series, the RP approach to partial nephrectomy was associated with shorter LOS and equivalent rates of collectingsystem entry, positive margins, conversion to alternate procedures, and complications. The EBL was lower in the RP approach overall and in patients without complications but was equivalent to that of the TP approach in patients with complications. Although RP patients had fewer complications, their complications were more severe and required more specialized care than the complications in TP patients.

Variations in surgical outcomes between these groups likely reflect the characteristics of the treated lesion as well as of the surgical procedure (e.g., surgeon skill, limitations of the operative procedure). Preoperative and postoperative renal function, renal ischemia times, and positive-margin rates were similar in the two groups, suggesting that selection of a particular approach impacts dissection and exposure of the kidney rather than resection of the mass. The extensive mobilization of the colon and kidney in the TP approach (especially for posterior

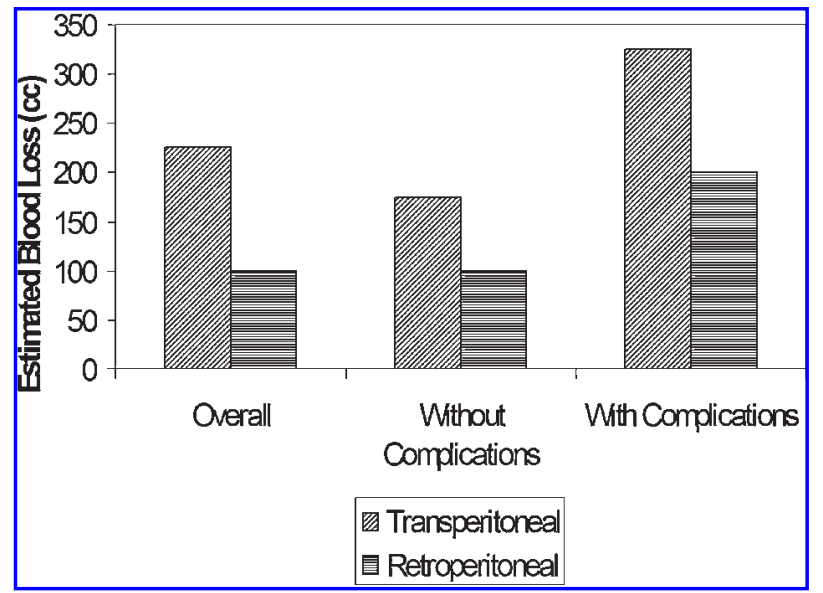

FIG. 3. Median estimated blood loss generally is lower with $\mathrm{RP}$ approach, although this difference is minimized in patients with perioperative complications. masses ${ }^{12-14,18}$ ) likely accounts for the longer median LOS, longer operative time (but similar renal ischemic time), and predominance of postoperative ileus in TP patients. Although the $\mathrm{RP}$ route is more direct, unfamiliar anatomy may hamper the inexperienced surgeon; identification of anatomic landmarks may be improved and operating time shortened with careful and adequate balloon dissection of the retroperitoneal space early in the procedure. ${ }^{20}$

The skills necessary for TP laparoscopic nephrectomy are rapidly acquired, ${ }^{9,21,22}$ and decreasing operative times with the $\mathrm{RP}$ approach suggest a learning curve ${ }^{23}$ for this procedure as well. However, RP partial nephrectomies are made more complex, not only by the advanced laparoscopic techniques (e.g., intracorporeal suturing) needed for partial nephrectomy, but also by the limited working space (often requiring specialized instruments) ${ }^{13,24,25}$ and comparatively unfamiliar retroperitoneal anatomy. ${ }^{14,15,20}$ Our data and recently published work ${ }^{13}$ suggest that experienced surgeons can overcome these limitations to achieve statistically equivalent outcomes with RP and TP partial nephrectomies. At our institution, RP nephrectomy was introduced in August 1997, TP partial nephrectomy in November 1998, and RP partial nephrectomy in February 2001. The present analysis excludes three patients operated on prior to 2003 to avoid the confounding effect of surgeon inexperience with RP partial nephrectomy.

Of particular interest was the difference in the complication rates in our TP and RP patients. Excluding retroperitoneal hemorrhage (the most common complication in both groups), more complications (e.g., prolonged ileus, readmission for pain control) in TP patients could be attributed to dissection technique. Patients having RP surgery may require fewer postoperative resources owing to the limited bowel dissection and lower likelihood of gastrointestinal complications. An RP nephrectomy may be especially useful in patients with prior abdominal surgery or radiation, as convalescence and recovery of bowel function are not prolonged with this approach. ${ }^{14}$

The most severe complications in our series (e.g., atrial fibrillation, renal-vein avulsion) occurred in the only patient with an intraoperative complication: avulsion of the renal vein and severe hemorrhage during initial balloon expansion of the retroperitoneum necessitating two-stage radical nephrectomy. 
On retrospective review, this event likely was related to positioning of the balloon medial to the lower pole of the kidney within the retroperitoneal space, which enabled the inflation force to be directed primarily at the renal vein rather than the kidney. Postoperatively, the patient had a prolonged ICU course. Although such severe complications certainly are uncommon, the sequelae in this patient underscore the technical considerations during retroperitoneal surgery that may make management of some complications more difficult. ${ }^{13-15} \mathrm{~A}$ hand or new instrument may be inserted easily in the TP approach, whereas limited space and visibility during RP procedures limits additional port access and other options. ${ }^{15}$ The hand-assisted RP approach ${ }^{17,26}$ has not been employed at our institution. Intrarenal tumor location is an important factor in the decision to pursue a TP or an RP approach ${ }^{12,13}$; unfortunately, the small sample size in this study does not allow a meaningful analysis of differential complication rates with the two surgical approaches.

At least some complications may be secondary to limitations of the RP procedure, as anatomic considerations prompt the majority of conversions to open procedures. ${ }^{23}$ Although our conversion rates are equivalent in TP and RP patients and are consistent with published data, ${ }^{14,23}$ the potential benefit of the RP approach with respect to EBL was lost in patients with complications. We caution surgeons to be aware of the challenges and limitations of managing complications in the retroperitoneal space.

Our research has several limitations. The small sample size will capably detect only the largest differences between the RP and TP groups, and retrospective medical record review cannot capture subjective intraoperative findings such as tissue quality and difficulty of dissection, nor characterize the severity of complications with broad qualitative variations (e.g., ileus). Moreover, we did not specifically evaluate lesion location within the kidney as an independent variable. As tumor location is the primary factor we used in selecting the approach, and none of our patients was selected for RP approach on the basis of factors such as prior intra-abdominal surgery, the distribution of tumor locations in the two groups was quite different. However, the most direct route to the renal mass should facilitate dissection and minimize manipulation, thereby limiting complications, so we believe that our comparison of RP and $\mathrm{TP}$ patients is appropriate despite this limitation.

\section{CONCLUSIONS}

There exists a great demand for minimally invasive therapies for the management of renal masses. Our experience suggests that minimally invasive partial nephrectomy can be performed transperitoneally or retroperitoneally with equivalent outcomes in carefully selected patients. The RP approach may avoid extensive dissection and decrease morbidity and LOS without compromising the quality of tumor resection during partial nephrectomy. Surgical outcomes in $\mathrm{RP}$ procedures can be improved with appreciation for anatomic considerations and with increasing surgeon experience. These data reflect our preliminary results with RP partial nephrectomy; further experience, ideally in a randomized trial involving masses amenable to either approach, is needed to evaluate long-term outcomes.

\section{REFERENCES}

1. Jayson M, Sanders H. Increased incidence of serendipitously discovered renal cell carcinoma. Urology 1998;51:203.

2. Hafez KS, Fergany AM, Novick AC. Nephron sparing surgery for localized renal cell carcinoma: Impact of tumor size on patient survival, tumor recurrence and TNM staging. J Urol 1999;162:1930.

3. Patard J-J, Shvarts O, Lam JS, et al. Safety and efficacy of partial nephrectomy for all $\mathrm{T} 1$ tumors based on an international multicenter experience. J Urol 2004;171:2181.

4. Makhoul B, Taille A de la, Vordos D, et al. Laparoscopic radical nephrectomy for $\mathrm{T} 1$ renal cancer: The gold standard? A comparison of laparoscopic vs open nephrectomy. BJU Int 2004;93:67.

5. Gill IS, Matin SF, Desai MM, et al. Comparative analysis of laparoscopic versus open partial nephrectomy for renal tumors in 200 patients. J Urol 2003;170:64

6. Dunn MD, Portis AJ, Shalhav AL, et al. Laparoscopic versus open radical nephrectomy: A 9-year experience. J Urol 2000;164:1153.

7. Meraney AM, Gill IS. Financial analysis of open versus laparoscopic radical nephrectomy and nephroureterectomy. J Urol 2002; $167: 1757$

8. Iwamura M, Tsumura H, Matsu D, et al. Port site recurrence of renal cell carcinoma following retroperitoneoscopic radical nephrectomy with manual extraction without using entrapment sac or wound protector. J Urol 2004;171:1234.

9. Chan DY, Cadeddu JA, Jarrett TW, et al. Laparoscopic radical nephrectomy: Cancer control for renal cell carcinoma. J Urol 2001; 166:2095.

10. Cadeddu JA, Ono Y, Clayman RV, et al. Laparoscopic nephrectomy for renal cell cancer: Evaluation of efficacy and safety: A multicenter experience. Urology 1998;72:773.

11. Saika T, Ono Y, Hattori R, et al. Long-term outcome of laparoscopic radical nephrectomy for pathologic $\mathrm{T} 1$ renal cell carcinoma. Urology 2003;62:1018.

12. Gill IS, Colombo JR Jr, Frank I, et al. Laparoscopic partial nephrectomy for hilar tumors. J Urol 2005;174:850.

13. Ng CS, Gill IS, Steinberg AP, et al. Transperitoneal versus retroperitoneal laparoscopic partial nephrectomy: Patient selection and perioperative outcomes. J Urol 2005;174:846.

14. Viterbo R, Greenberg RE, Al-Saleem T, Uzzo RG. Prior abdominal surgery and radiation do not complicate the retroperitoneoscopic approach to the kidney or adrenal gland. J Urol 2005;174: 448.

15. Meraney AM, Samee, AA-E, Gill IS. Vascular and bowel complications during retroperitoneal laparoscopic surgery. J Urol 2002;168:1941

16. Bishoff JT, Allaf ME, Kirkels W, Moore RG, Kavoussi LR, Schroder F. Laparoscopic bowel injury: Incidence and clinical presentation. J Urol 1999;161:887.

17. Wadstrom J. Hand-assisted retroperitoneoscopic live donor nephrectomy: Experience from the first 75 consecutive cases. Transplantation 2005;80:1060.

18. Takeda M, Go H, Watanabe R, et al. Retroperitoneal laparoscopic adrenalectomy for functioning adrenal tumors: Comparison with conventional transperitoneal laparoscopic adrenalectomy. J Urol 1997; 157:19.

19. Matin SF, Gill IS. Laparoscopic radical nephrectomy: Retroperitoneal versus transperitoneal approach. Curr Urol Rep 2002;3:164.

20. Sung GT, Gill IS. Anatomic landmarks and time management during retroperitoneoscopic radical nephrectomy. J Endourol 2002;16: 165. 
21. Vallancien G, Cathelineau X, Baumert H, et al. Complications of transperitoneal laparoscopic surgery in urology: Review of 1,311 procedures at a single center. J Urol 2002;168:23.

22. Hollenbeck BK, Seifman BD, Wolf JS Jr. Clinical skills acquisition for hand-assisted laparoscopic donor nephrectomy. J Urol 2004; $171: 35$.

23. Rassweiler JJ, Seemann O, Frede T, Henkel TO, Alken P. Retroperitoneoscopy: Experience with 200 cases. J Urol 1998;160:1265.

24. Ramani AP, Desai MM, Steinberg AP, et al. Complications of laparoscopic partial nephrectomy in 200 cases. J Urol 2005;173:42.

25. Johnston WK III, Montgomery JS, Wolf JS Jr. Retroperitoneoscopic radical and partial nephrectomy in the patient with cirrhosis. J Urol 2005;173:1094.

26. Sato Y, Tanda H, Kato S, et al. 100-Minute retroperitoneoscopic radical nephrectomy: A new retroperitoneal approach with a hand assisted method. J Urol 2005; 174:53.
Address reprint requests to: J. Stuart Wolf, Jr., M.D.

Dept. of Urology

University of Michigan

1500 East Medical Center Drive, TC 3875

Ann Arbor, MI 48109

E-mail:wolfs@umich.edu

\section{ABBREVIATIONS USED}

ANOVA $=$ analysis of variance; $\mathrm{EBL}=$ estimated blood loss; ICU = intensive care unit; LOS = length of stay; RP = retroperitoneal; $\mathrm{TP}=$ transperitoneal. 
This article has been cited by:

1. Henry Sun Sien Ho, Reinhard Peschel, Richard Neururer, Hannes Steiner, Christian Schwentner, Georg Bartsch . 2008. Another Novel Application of Hem-o-Lok Clips for Transient Vascular Occlusion in Robot-Assisted Laparoscopic Partial Nephrectomy: An Alternative to Laparoscopic Bulldog and Satinsky ClampsAnother Novel Application of Hem-o-Lok Clips for Transient Vascular Occlusion in Robot-Assisted Laparoscopic Partial Nephrectomy: An Alternative to Laparoscopic Bulldog and Satinsky Clamps. Journal of Endourology 22:8, 1677-1680. [Abstract] [PDF] [PDF Plus] 